\title{
The Caterpillar Fungus Cordyceps Sinensis as a Natural Source of Bioactive Compounds
}

\author{
Mouhanad AL ALI \\ (Institute of Bioproduct Development, Universiti Teknologi Malaysia, Malaysia)
}

\begin{abstract}
Cordyceps fungi have attracted many mycologists since their discovery because of their peculiar characteristics of fruiting on their host invertebrate animals. Cordyceps fungi, which parasitize insects and change into fruiting bodies, are rich sources of biologically active compounds that are implicated for improvement in health and for prevention and treatment of various diseases. They are considered to be some of the more promising medicinal mushrooms. Cordyceps sinensis has been noted for its potential for medicinal use and hence extensive pharmaceutical studies have been made. In this review I compile available data on the characteristics of Cordyceps sinensis that appear to be effective as a natural source of bioactive compounds.

Keywords - bioactive compounds, Cordyceps sinensis, caterpillar fungus, medicinal mushrooms.
\end{abstract}

\section{INTRODUCATION}

In recent years, the interest in mushrooms as a dietary fiber or healthy food has increased. In addition, they have received significant attention from medical and pharmacological researchers as rich sources of biologically active compounds[1]. The "Chinese caterpillar fungus", that is, "Vegetable wasps and plant worms" is one of the traditional medicinal mushrooms belonging to entomopathogenic fungi. The fungus invades and proliferates within a specific insect, eventually killing the host. After passing the winter inside the host, it forms a fruiting body on the surface of the host insect's cadaver next summer. Because there are many kinds of host insects, there are many known species of Chinese caterpillar fungi, which belong to many different genera such as Cordyceps, Torrubiella and Paecilomyces. The most popular Cordyceps species, Cordyceps sinensis, which is a parasite on the larvae of Hepialus armoricanus, has long been used to promote longevity, relieve exhaustion and treat numerous diseases as Chinese traditional medicines [2], [3].

Recent studies have demonstrated that various species possess multiple pharmacological actions, to be specific, anti-tumor, anti-microbial, anti-inflammatory and immunomodulatory effects [4],[5]. Furthermore, a variety of the effective chemical constituents including cordycepin, ergosterol, myriocin and polysaccharides have been isolated from various species [6],[7]. Particularly, cordycepin (3'-deoxyadenosine) is a nucleoside analogue, which has a broad spectrum of biological activity. In this review I compile available data on the characteristics of Cordyceps sinensis that appear to be effective as a natural source of bioactive compounds.

\section{CATERPILLAR HOSTS}

Though the Tibetan name for C. sinensis is yartsa gunbu ("summer-grass, winter-worm"), it is commonly referred to simply as bu ("worm" or "insect"). The full name describes the main life stages of Cordyceps sinensis: its infected caterpillar host passes the winter (gun) in the soil as a "worm"; then in early summer (yar) the mushroom or "grass" (tsa) emerges above ground. The word tsa is usually translated as grass, but can also be used to denote some mushrooms.

Cordyceps sinensis parasitizes various grass rootboring Thitarodes (Hepialus) caterpillars, which hatch as "ghost moths" when not preempted by Cordyceps. Thirty of the nearly 40 species of Thitarodes known from the Tibetan Plateau can be infected by Cordyceps sinensis [8]. Many of the regional differences in size of the Cordyceps mushroom are probably caused by size differences in the host species. While the normal reproductive cycle for Thitarodes species takes up to five years, most of the life cycle is lived as a caterpillar; the whitish adult moth lives for only a few days in order to mate.

Uninfected larvae typically hibernate deeper in the soil than infected ones; apparently the fungus is able to force the infected host to situate itself nearer the soil surface in a position more favorable to its fruiting. The hyphae of the mycelium develop inside the body of the insect, first feeding on less vital parts before taking over the complete organism. Eventually the insect is completely mummified and emptied of nutrients, and all that remains is the exoskeleton filled and coated with Cordyceps mycelium. In spring, the mushroom (also known as the fruiting body or stroma) develops out of the head of the exoskeleton just above the eyes. The slender, brown, club-shaped fruiting body then emerges from the ground, reaching a total length of $8-15 \mathrm{~cm}$. It is typically nearly twice as long as its caterpillar host, but in rare cases can be up to four times as long. 


\section{DISTRIBUTION}

Cordyceps sinensis is endemic to the grasslands and shrublands of central Asia and in particular the Tibetan Plateau at altitudes of 3,000 to 5,000 m. The grasslands providing habitat for Thitarodes ghost moths and thus for Cordyceps sinensis consist predominantly of sedges (Kobresia spp.), which can cover up to $80-90 \%$ of the subalpine grasslands [9].Thitarodes moths prefer to feed on young roots of plant species of the families Polygonaceae, Fabaceae, Cyperaceae (including Kobresia), Poaceae, and Liliaceae [8].

Cordyceps sinensis is also found in some mountainous areas of southwestern Sichuan and northern Yunnan that are inhabited by other ethnic minorities. Reports of it from the central and eastern Himalayas (Nepal, Bhutan, and India) are difficult to verify because of confusion with other Himalayan species, such as $C$. nepalensis [10]. C.sinensis has also been reported from Tian Shan and Altai Shan in Xinjiang province of northwestern China [10].

Cordyceps sinensis is considered to be one rare and valuable medicinal fungus for preventing and treating diverse chronic diseases, and its quality and price vary hugely with different producing areas. Unfortunately, it is difficult to discriminate geographical origin, owing to uncertainties of existing methods by a simple visual or an odor check [11]. In recent years, the researchers were trying to discriminate the geographical origin of Cordyceps sinensis by a nucleic acids based method such as amplified fragment length polymorphism (AFLP), however, the result are not pleasant. Thus, it is necessary to develop new methods and search for sensitive indicators to detect geographical origin. Cordyceps sinensis occurs on the Tibetan Plateau and its adjacent high-altitude areas characterized by low ambient temperatures [12],[13].And its lipid composition may be a sensitive marker reflective of its living environment. Lately, it has been found that the fatty acid composition in lipids may be used to access geographic origin. The fatty acid profile are suggested to be one potential indicator for determination of geographical origin. [14].

\section{IMPORTANCE OF CORDYCEPS SINENSIS}

Cordyceps sinensis has been used in China for more than 2000 years as one rare health food or medicinal fungi tontreat diverse chronic diseases [15],[17].with beneficial effects on renal and hepatic function [18] as well as immunomodulation-related anti-tumour activities [19]. Modern pharmacological studies have shown that C.sinensis possesses wide biological activities, e.g., anti-oxidation, immunopotentiation, antitumorigenesis, anti inflammation, inhibiting hepatic fibrosis, stimulation of testosterone biosynthesis, enhancement of splenocyte proliferation, myocardial mitochondrial ATP generation and anti-hyperglycemia [15],[16],[19],[20]. Previous studies suggest that Cordyceps.sinensis contains a variety of bioactive components, mainly polysaccharides, proteins, lipids, nucleosides and bases [21],[22]. C.sinensis as one rare and precious herbal medicine has already been marketed in many countries or districts, especially mainland China, Hong Kong, Taiwan, Japan and Korea.

\section{THE BIOLOGICAL ACTIVITY OF CORDYCEPIN}

cordycepin (3_-deoxyadenosine) is a nucleoside analogue, which is one of the most versatile metabolites of cordyceps sinensis due to its broad spectrum of biological activity. It has been reported that cordycepin is intracellularly converted into its 5'-mono, di and triphosphates and they inhibit the activity of ribose-phosphate pyrophosphokinase and 5- phosphoribosyl 1-pyrophosphate amidotransferase in the de novo biosynthesis of purines and/or the synthesis of nucleic acids, causing the anti-tumor, anti-metastatic and antimicrobial effects [23-24].In addition, the anti-leukemic activity of cordycepin combined with an adenosine deaminase inhibitor and the inhibitory effect of its analogues of $2^{\prime}, 5^{\prime}$-oligoadenylate on human immunodeficiency virus infection have also been displayed [25], [26].

\section{CONCLUSION}

Cordyceps is one of the target genera for modern mycological studies. Among them Cordyceps sinensis is the most famous but poorly defined species because the fungus is endemic in districted regions of east Eurasia. The caterpillar fungus, is parasitic on the larvae of Lepidoptera and the host insects have been reported as members of Hepialidae (Lepidoptera, Exoporia; the bat moths), which are distributed in cool weather conditions. They have been variously described. But identification of the host insects by larvae stage is difficult and identification of the exact host species awaits future determination. It is very interesting that the fungus is able to use plural genera or species as the host insect.

The major bioactive metabolites found in medicinal mushrooms can be broadly categorized as high molecular weight metabolites and low molecular weight metabolites. High molecular weight metabolites such as polysaccharides, protein polysaccharides and fungal immunomodulatory proteins provide anti-cancer activity mainly thorough the activation of the immune system. Mushrooms may produce large numbers of low molecular weight compounds that not only activate the immune system but also control the cellular transduction pathways responsible for cancer development. 
Medicinal mushrooms are gifts from nature that contain biologically active metabolites which can be used as support remedies for cancer treatments. Additional studies of the activities and mechanisms of action of these metabolites are needed so to develop them as potent anti-cancer drugs. New methods and techniques integrated with biotechnology and other relevant disciplines are also required.

\section{REFERENCES}

[1] Wasser SP. "Medicinal mushrooms as a source of antitumor and immunomodulating polysaccharides". Appl Microbiol Biotechnol 2002;60: 258-74.

[2] Pegler DN, Yao Y-J, Li Y. “The Chinese "caterpillar fungus"”. Mycologist 1994;8:3-5.

[3] Buenz EJ, Bauer BA, Osmundson TW, Motley TJ. "The traditional Chinese medicine Cordyceps sinensis and its effects on apoptotic homeostasis". J Ethnopharmacol 2005;96:19-29.

[4] Mizuno T. "Medicinal effects and utilization of Cordyceps (Fr.) Link (ascomycetes) and Isaria Fr. (mitosporic fungi) Chinese caterpillar fungi”, “Tochukaso" (review). Int J Med Mushr 1999;1:251-61.

[5] Ng TB, Wang HX. "Pharmacological actions of Cordyceps, a prized folk medicine". J Pharm Pharmacol 2005;57:1509-19.

[6] Cunningham KG, Hutchinson SA, Manson W, Spring FS. "Cordycepin, ametabolic product from cultures of Cordyceps militaris" (Linn) Link. Part I. Isolation and characterization. J Chem Soc 1951:2299-300.

[7] Fujita T, Inoue K, Yamamoto S, Ikumoto T, Sasaki S, Toyama R, et al." Fungal metabolites II, Apotent immunosuppressive activity found in Isaria sinclairii metabolite". J Antibiot 1994;47:208-15.

[8] Chen, S. J., D. H. Yin, L. Li, X. Zha, J. H. Shuen, and C. Zhama. 2000. "Resources and Distribution of Cordyceps sinensis in Naqu Tibet". Zhong Yaocai 2311:673-675, (Chinese,English abstract).

[9] Wu, N. 1997. "Rangeland Resources and Conditions in Western Sichuan". Pages 23-40 in: D. J.

[10] Zang, M. and N. Kinjo. 1998. "Notes on the Alpine Cordyceps of China and Nearby Nations". Mycotaxon 66:215-229.

[11] Pan XY. "Identification of Cordyceps sinensis by experience". China Pharmacist 2006;9:380-1.

[12] Yang DR, Li CD, Shu C, Yang YX.”Studies on the Chinese species of the genus Hepialus and their geographical distribution.” Acta Entomol Sin 1996;39:413-22.

[13] Chen D, Yuan JP, Xu SP, Zhou XG, Zhang Y, Xu XM, et al.” Stable carbon isotope evidence for tracing the diet of the host Hepialus larva of Cordyceps sinensis in the Tibetan Plateau". Sci China (D) 2009;52:655-9.

[14] Lian-Xian Guo, Xiao-Ming Xu, Chou-Fei Wu, Li Lin, Shi-Chun Zou, Tian-Gang Luan, Jian-Ping Yuan, Jiang-Hai Wang.” Fatty acid composition of lipids in wild Cordyceps sinensis from major habitats in China" Biomedicine \& Preventive Nutrition. Feb 2012

[15] Yu HM, Wang BS, Huang SC, Duh PD. "Comparison of protective effects between cultured Cordyceps militaris and natural Cordyceps sinensis against oxidative damage". J Agric Food Chem 2006;54:3132-8.

[16] Zhang X, Liu YK, Shen W, Shen DM." Dynamical influence of Cordyceps sinensis on the activity of hepatic insulinase of experimental liver cirrhosis". Hepatobiliary Pancreat Dis Int 2004;3:99-101.

[17] Zhu JS, Halpern GM, Jones K. "The scientific rediscovery of an ancient Chi-nese herbal medicine": Cordyceps sinensis: part I. J Altern Complement Med 1998;4:289-303.

[18] Wojcikowski K, Johnson DW, Gobe G. "Medicinal herbal extracts - renal friend or foe?" Part one: the toxicities of medicinal herbs. Nephrology (Carlton) 2004;9:313-8.

[19] Paterson RR. "Cordyceps: a traditional Chinese medicine and another fungal therapeutic biofactory?" Phytochemistry 2008;69:1469-95.

[20] Zhang M, Cui SW, Cheung PCK, Wang Q. "Antitumor polysaccharides from mushrooms: a review on their isolation process, structural characteristics and antitumor activity". Trends Food Sci Tech 2007;18:4-19.

[21] Li SP, Li P, Lai CM, Gong YX, Kan KK, Dong TT, et al. "Simultaneous determination of ergosterol, nucleosides and their bases from natural and cultured Cordyceps by pressurised liquid extraction and high-performance liquid chromatography". J Chromatogr A 2004; 1036:239-43.

[22] Yuan JP, Wang JH, Liu X, Kuang HC, Zhao SY. "Simultaneous determination of free ergosterol and ergosteryl esters in Cordyceps sinensis by HPLC". Food Chem.

[23] Overgaard-Hansen K. "The inhibition of 5-phosphoribosyl-1-pyrophosphate formation by cordycepin triphosphate in extracts of Ehrlich ascites tumor cells". Biochim Biophys Acta 1964;80:504-7.

[24] Nakamura K, Konoha K, Yoshikawa N, Yamaguchi Y, Kagota S, Shinozuka K, et al. “Effect of cordycepin (3' -deoxyadenosine) on hematogenic lung metastatic model mice". In vivo 2005;19:137-42.

[25] Kodama EN, McCaffrey RP, Yusa K, Mitsuya H.” Antileukemic activity and mechanism of action of cordycepin against terminal deoxynucleotidyl transferase-positive (TdT+) leukemic cells". Biochem Pharmacol 2000;59:273-81.

[26] Muller WE, Weiler BE, Charubala R, Pfleiderer W, Leserman L, Sobol RW, et al." Cordycepin analogues of 2',5'-oligoadenylate inhibit human immunodeficiency virus infection via inhibition of reverse transcriptase". Biochemistry 1991;30:2027-33. 